\title{
Gestión de TI, Contratación Pública Electrónica: Equipamiento Médico y su incidencia en Instituciones de Salud IESS.
}

\section{IT Management, Electronic Public Procurement: Medical Equipment and its incidence in Health Institutions IESS}

DOI: $10.46932 / \mathrm{sfjdv2n2-196}$

Received in: March 1st, 2021

Accepted in: May 30th, 2021

\author{
Mgs. Mauricio Prado Ortega \\ Magister en Educación Superior, Magister en Salud para el Desarrollo Local \\ Universidad Técnica de Machala \\ Urbanización Santa Inés Mz F villa 18AB, Machala-Ecuador \\ E-mail:mprado@utmachala.edu.ec \\ Mgs. Héctor Carvajal Romero \\ Magíster en Administración de Empresas \\ Universidad Técnica de Machala \\ Urbanización Santa Inés Mz F villa 23B, Machala-Ecuador \\ E-mail: hcarvajal@utmachala.edu.ec \\ Mgs. Harry Vite Cevallos \\ Maestría en Sistemas de Información Gerencial \\ Universidad Técnica de Machala \\ Guayacanes y Tercera Diagonal, Machala-Ecuador \\ hvite@utmachala.edu.ec \\ E-mail: Machala, Ecuador \\ Mgs. Hayne Jara Castro \\ Magister en Gerencia de Salud para el Desarrollo Local \\ Universidad Técnica de Machala \\ Urbanización Ciudad Verde, Machala-Ecuador \\ E-mail: hjara@utmachala.edu.ec
}

\section{RESUMEN}

A partir de la implementación del portal de compras públicas en el año 2008 como herramienta tecnológica de contratación el Estado Ecuatoriano agilizó la adquisición de equipamiento médico a las instituciones en el ámbito del sector salud como ejemplo el Hospital del IESS de Machala y mejorar la atención en áreas como Fisioterapia, Traumatología, Medicina Familiar, Medicina General y otras dependencias. El desafío era adquirir los equipos médicos a través del portal de compras públicas y contemplados en el POA Institucional, hasta la actualidad es obligatorio utilizar esta herramienta publica electrónica bajo las diferentes modalidades de contratación según la LOSNCP. La población afiliada, jubilados y otros beneficiados necesitan a diario estos servicios y corresponde analizar la administración y gestión que realizan las autoridades provinciales del IESS para que esto se realice en el tiempo oportuno. La presente investigación presenta una descripción de la complejidad de los equipos médicos adquiridos por esta casa de salud y los beneficios que derivaron a que varios servicios sean más productivos en los años 2011 al 
2013 y con el buen manejo del Portal de Compras Públicas facilitan y optimizan la tarea de contratación brindando servicios de salud de "calidad y calidez".

Palabras clave: Tecnología, Contratación Pública Electrónica, Salud Pública.

\section{ABSTRACT}

Starting with the implementation of the public procurement portal in 2008 as a technological contracting tool, the Ecuadorian State expedited the acquisition of medical equipment from institutions in the health sector, such as the IESS Hospital in Machala and improving care in areas such as Physiotherapy, Traumatology, Family Medicine, General Medicine and other dependencies. The challenge was to acquire the medical equipment through the public procurement portal and contemplated in the Institutional POA, until now it is mandatory to use this electronic public tool under the different contracting modalities according to the LOSNCP. The affiliated population, retirees and other beneficiaries need these services on a daily basis and it is necessary to analyze the administration and management carried out by the provincial authorities of the IESS so that this is carried out in a timely manner. The present research presents a description of the complexity of the medical equipment acquired by this health house and the benefits that derived from various services being more productive in the years 2011 to 2013 and with the good management of the Public Procurement Portal they facilitate and optimize the task of contracting providing health services of "quality and warmth"

Keywords: Technology, Electronic Public Procurement, Public Health.

\section{INTRODUCCIÓN}

La complejidad gubernamental a nivel nacional y local en temas de contratación pública del sector de la salud pública en el Ecuador ha sufrido una importante transformación de procesos apoyadas por la gestión TI y la aparición del término gobierno electrónico. Uno de los centros de salud en la ciudad de Machala como es el IESS Hospital General Machala Nivel II, hace una década atrás inicio el reto de adquirir por medio de la herramienta del portal de compras públicas la adquisición de equipos médicos, instrumental e insumos que hasta la actualidad han permitido brindar tratamientos en áreas y servicios importantes como Rehabilitación y Terapia Física, Quirófanos, la primera UCI de adultos, UCI infantil, Cardiología, generando una facturación de estos servicios por encima de los niveles de productividad de su personal médico y contribución de equipos de trabajos administrativos capacitados en manejo de aplicaciones y herramientas gubernamentales. La población de afiliados a la seguridad social en el año 2010 fue de 60.078 personas en la provincia de El Oro y en 2020 a pesar del COVID 19 son cerca de 85.000 personas entre afiliados, pensionistas de montepío, voluntarios, seguro campesino y jubilados, sin contar la atención a cónyuges, amas de casa, niños y la atención a la RED del MSP, ISSFA, ISSPOL. Se toma como referencia histórica a nivel nacional la cobertura de varios seguros existentes en los diferentes estamentos públicos como se visualiza en la tabla 1. 
Tabla 1. Cuadro IV de cobertura de la seguridad social, Ecuador 2010

\begin{tabular}{lrrrrrr}
\hline Seguro & Cotizantes & \multicolumn{1}{c}{ Jubilado } & Montepío & Dependientes & \multicolumn{1}{c}{ Total } & Porcentaje \\
\hline IESS & $1,826.911$ & 187.911 & 90.060 & 545.685 & $2,650.567$ & 65,70 \\
SSC & 227.694 & 34.729 & - & 689.723 & 952.146 & 23,60 \\
ISSFA & 37.719 & 25.379 & 11.576 & 148.384 & 223.058 & 5,50 \\
ISSPOL & 39.286 & 10.450 & 6.616 & 154.179 & 210.531 & 5,20 \\
\hline Total & $2,131.610$ & 258.469 & 108.252 & $1,537.971$ & 4,036302 & 100.00 \\
\hline
\end{tabular}

Fuente: Quito: Ministerio de Coordinación de Desarrollo Social, 2010.

Por tanto, emplear y administrar el dinero del sector público no es nada fácil, la contratación pública por medios electrónicos forzó a todas las entidades gubernamentales a un cambio de paradigmas justamente para evitar suspicacias de la contratación pública tradicional. Lo que se realiza en la actualidad por medio del Portal de Compras Públicas en el Ecuador es un mecanismo electrónico, es el instrumento por medio del cual la administración estatal, utilizando en lo esencial medios electrónicos, busca satisfacer sus necesidades de contratación (Laguado, 2004).

En este contexto la Ley Orgánica del Sistema Nacional de Contratación Pública, publicada el 22 de julio de 2008, dentro de la transición que vivió el país durante el proceso constituyente, la cual viene a articular los ya existentes procesos de contratación y adquisición de bienes y servicios, pero que pone su mayor énfasis en la planificación, organización, ahorro de recursos, y transparencia a la hora de ejecutarlos a través de un sistema ágil y sobre todo que aprovecha de manera satisfactoria las herramientas desarrolladas por las TIC; cuyo ámbito de actuación engloba a todos los organismos y dependencias del Estado y que se lo desarrolla en los ocho numerales del artículo primero del mencionado cuerpo legal (Moscoso, 2014).

El Sistema Nacional de Contratación Pública (SERCOP) a través del Sistema Oficial de Contratación del Ecuador, ha enfocado sus esfuerzos en garantizar la calidad del gasto público, así como la ejecución plena de los contratos y la aplicación efectiva de las normas contractuales, la transparencia y evitar la discrecionalidad, convertir la contratación pública en un elemento dinamizador de la producción nacional, estableciendo procesos para agilitar y simplificar las adquisiciones, siendo estos: catálogo electrónico, subasta inversa electrónica, menor cuantía, cotización, licitación, contratación directa, lista corta, concurso público y ferias inclusivas (Rivas \& Zambrano, 2019).

Es interesante conocer que la contratación pública sobre todo en el ámbito de la salud pública sea muy compleja, pues aglutina a otros sectores como administración, finanzas, jurisprudencia, estado, y tecnología. Para estos fines los profesionales de las ramas contables, financieras, económicas, del derecho y tecnológico, deben capacitarse y en eso las entidades públicas ahondan en recursos para el personal encargado de manejar el acceso al portal de compras. Por esta razón se realizan subprocesos desde subir el PAC, y subcomisiones encargadas de la realización de pliegos, etapas de negociación por cada proceso de adquisición y hasta administrar contratos. Por una parte, en cuanto a los aspectos de soporte a los 
procedimientos de contratación, se destaca la publicación por medios electrónicos de la información relativa a los contratos y la presentación, por vía electrónica, de las ofertas que realicen los distintos operadores (Delpiazzo, 2011). Ver figura 1.

Figura1. Interacción de Profesionales al momento de realizar adquisiciones en Salud

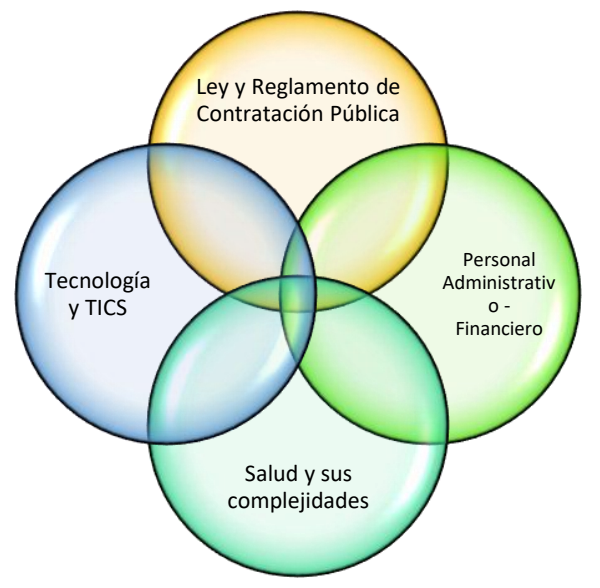

Con la creación de sistemas que permiten el manejo y el procesamiento de datos, fundamentales para distintos procedimientos administrativos, el mundo se inserta en la era del conocimiento, se ha convertido en una sociedad de la información. Parte importante de esta «sociedad» son los sistemas de información propiamente dichos y, por supuesto, Internet. Esta última es la herramienta clave para concretar transacciones con el Estado a través del nuevo sistema de compras del sector público. Pero es innegable que no todos los ciudadanos o los agentes económicos tienen acceso a computadoras y, menos aún, conexión a Internet (Ganga Contreras \& Águila Sánchez, 2006).

Definitivamente una gobernanza electrónica involucra una estructura tecnológica, que involucrar el uso de las TIC, así como el Internet que en nuestro país sigue creciendo. En la actualidad todos los procesos de contratación se realizan a través del portal de compras públicas obligatoriamente. En este contexto, la Gestión TI facilita las comunicaciones, el acceso a información (Internet) y a servicios sociales de educación, salud y gobierno electrónico, traen consigo profundas transformaciones sociales, económicas y culturales, que reflejan un aumento de la productividad y la aparición de economías de servicios virtuales, contribuyen al crecimiento económico, social, cultural, la modernización del Estado y la distribución con equidad, así como a desarrollar plataformas virtuales locales, nacionales y regionales de cooperación, para asegurar una participación eficiente en la economía digital (Barragan Martinez \& Guevara Viejo, 2016).

En lo que respecta al tema de la salud pública y el equipamiento médico, se debe tener muy en cuenta las especificaciones técnicas que elabore una comisión técnica integrada por médicos para que los profesionales encargados de la contratación puedan elaborar los pliegos y términos de contratación para 
llevar a cabo un proceso. Si esto no se realiza, los oferentes enviaran sus propuestas muy escuetas y es posible que califiquen en la fase de selección de la oferta. Todo esto parte de un listado de definiciones que precisan el alcance o significado de cada una de las locuciones que en él se utilizan; continúa con el ámbito de aplicación, excepciones, principios, publicación de información, publicación de avisos de las contrataciones que vayan a realizar las partes, las condiciones de participación, tipos de procedimientos de contratación: licitación pública, licitación selectiva, contratación directa, subasta electrónica; lista de uso múltiple; reglas para las especificaciones técnicas; pliegos de condiciones; plazos; negociaciones; reglas para la contratación directa; subasta electrónicas; tratamiento de las ofertas y adjudicación de los con tratos; transparencia de la información sobre contratación pública; divulgación de la información; procedimientos internos de revisión modificaciones y rectificaciones de la cobertura; participación de las micro, pequeñas y medianas empresas; cooperación; y la conformación de un subcomité sobre contratación pública (Aguirre Ribadeneira, 2016).

Como los procesos de contratación pública electrónica en el Ecuador desde el año 2008 son dinámicos, una de las modalidades más frecuentes que sigue utilizando la mayoría de hospitales es la subasta inversa electrónica y cotización. En el caso de la subasta inversa las bases son mucho más sencillas porque las especificaciones técnicas están predeterminadas en la ficha técnica y no hay factores de evaluación, en vista de que el proceso se define en función del precio. (Bossano Lomellini, 2011).

En vista de lo establecido para efectos del proceso de cotización en la LOSNCP en su artículo 50 manifiesta: Procedimientos de Cotización.- Este procedimiento, se utilizará en cualquiera de los siguientes casos: Si fuera imposible aplicar los procedimientos dinámicos previstos en el Capítulo II de este Título o, en el caso que una vez aplicados dichos procedimientos, éstos hubiesen sido declarados desiertos; siempre que el presupuesto referencial oscile entre 0,000002 y 0,000015 del Presupuesto Inicial del Estado del correspondiente ejercicio económico (Registro Oficial República del Ecuador, 2008). Ver figura 2 
Figura 2. Diagrama de aplicación de modalidades de procedimientos de contratación.

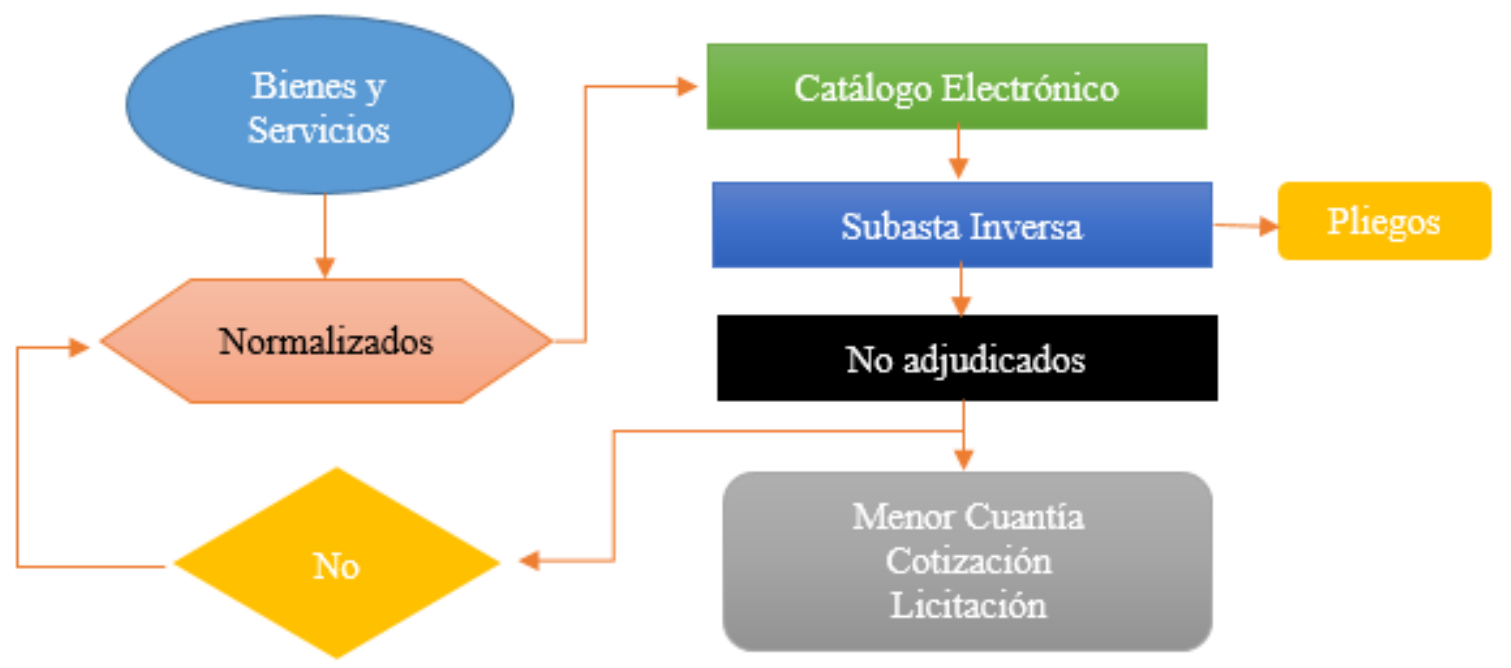

Los equipos médicos en la actualidad se encuentran normalizados, pero existen algunos equipos que necesariamente tienen características que se deben establecer en les especificaciones técnicas que los hacen únicos sin ser exclusivos. Esto es lo que se trata de prevenir para que los proveedores puedan participar en igualdad de condiciones. Sin embargo, es importante señalar que las comisiones técnicas buscan siempre lo mejor en calidad para sus pacientes cuando se trata de equipamiento médico, muchas veces este punto de la normalización se puede convertir en un acto motivado a ser corruptible. El ambiente normativo en que se desenvuelve la Contratación Pública en la región refleja el rol meramente burocrático que tradicionalmente se le ha atribuido, así como la situación de desconfianza general hacia los operadores que gestionan la Contratación Pública. Por otra parte, las cada vez más frecuentes críticas al desempeño de los sistemas de contrataciones públicas, aparecen como consecuencia de los reiterados fracasos de la gestión de adquisiciones en la producción de resultados de desarrollo a los que aquella debe estar subordinada. Esto comienza a respaldar un cambio en la percepción hacia la Contratación Pública, orientándola hacia un rol más estratégico, con mayor necesidad de flexibilidad, que permita el empleo de juicio profesional y habilidades gerenciales para la solución delas situaciones que la articulación adecuada de su gestión con los objetivos institucionales demanda (Chinea, 2013).

Por tanto, adquirir un equipo médico dista mucho de comprar otros bienes por su clara connotación de lo que representa en el ámbito de la salud, es necesario no solo considerar el costo beneficio, sino que implica todo un estudio que demandará infraestructura, personal operativo, insumos, tecnología, servicio técnico del fabricante y otros. Además, y de suma importancia, se debe demostrar estudios sobre la eficacia de los equipos médicos en el tratamiento de las diferentes patologías para determinados fines. Figura 3 
Figura 3. Esquema al considerar adquirir un equipo médico.
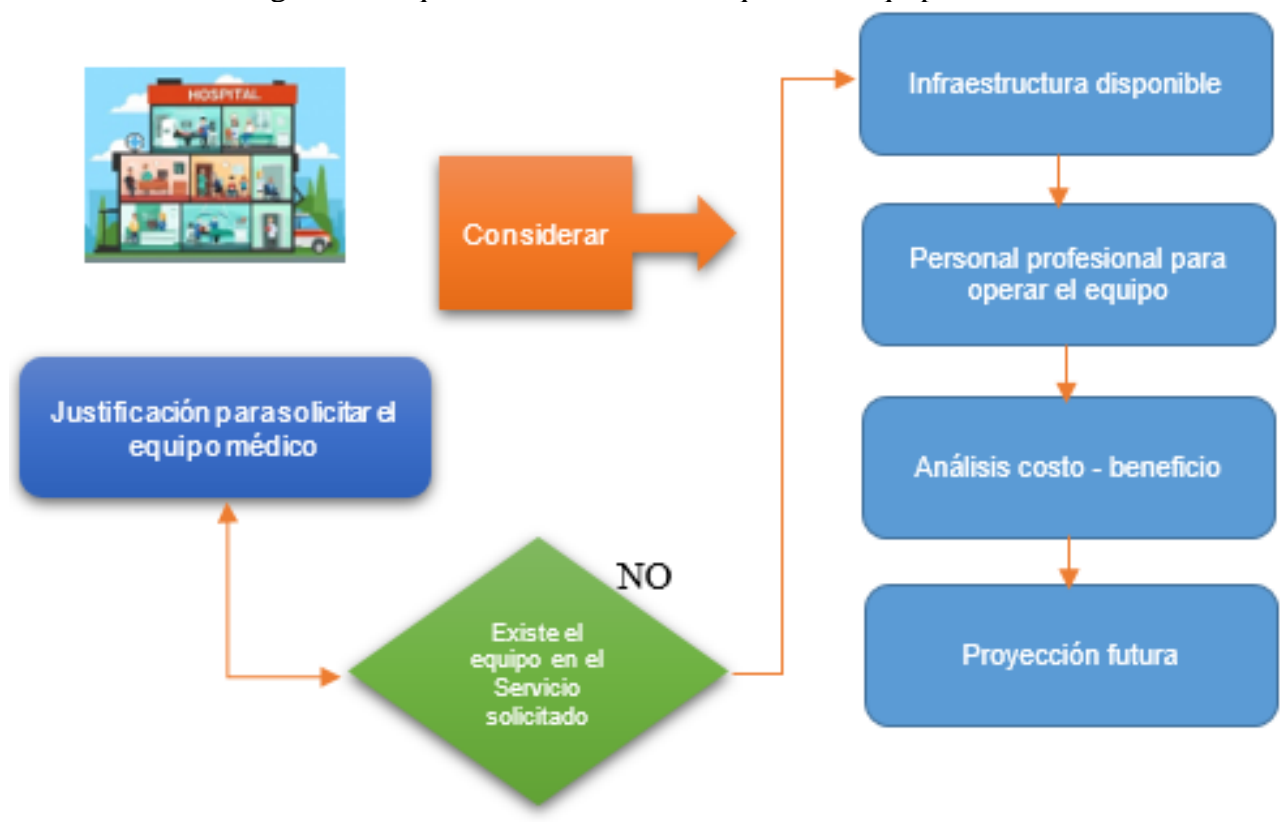

Otros requisitos que deben pasar estos aparatos sofisticados son los estándares de calidad. Estos los determinan transnacionales que tienen patentes y certificaciones bajo protocolos de calidad internacional, es así que los sellos que debe tener un equipo médico al momento de ser importado deben cumplir las normas impuestas por los fabricantes. Por tanto, si el equipo médico es europeo debe tener el logotipo de la comunidad europea que certifica su comercialización a nivel mundial. Los Estados Unidos y los demás países que componen América del Norte colocan su aval mediante las siglas FDA para sus fabricantes y si son realizados o fabricados en Asia estos llevan el logotipo de estándar asiático; México también tiene su propia norma NOM. Entre las normas más comunes de buenas prácticas de manufactura del fabricante que son aceptadas en Ecuador, tenemos:

- Cumplimiento de Normas ISO9001:2000, o la que aplique para la especialidad del equipo ofertado

- Cumplimiento de Normas CE en caso de equipo procedente de la Comunidad Económica Europea

- Certificación dela FDA510(k)para equipos procedentes de los Estados Unidos de América

Todas estas prácticas benefician al adquiriente quien tiene la oportunidad de devolver el equipo médico por desperfectos o por no cumplir una de esta normas o certificación de calidad internacionales. Por ejemplo, UL es una norma que proporciona soluciones para la certificación y realización de ensayos en Norteamérica, América del Sur, Oriente Medio, África, Asia y Australia Figura 4. 
Figura 4. Vista de diferentes certificaciones de un equipo médico.

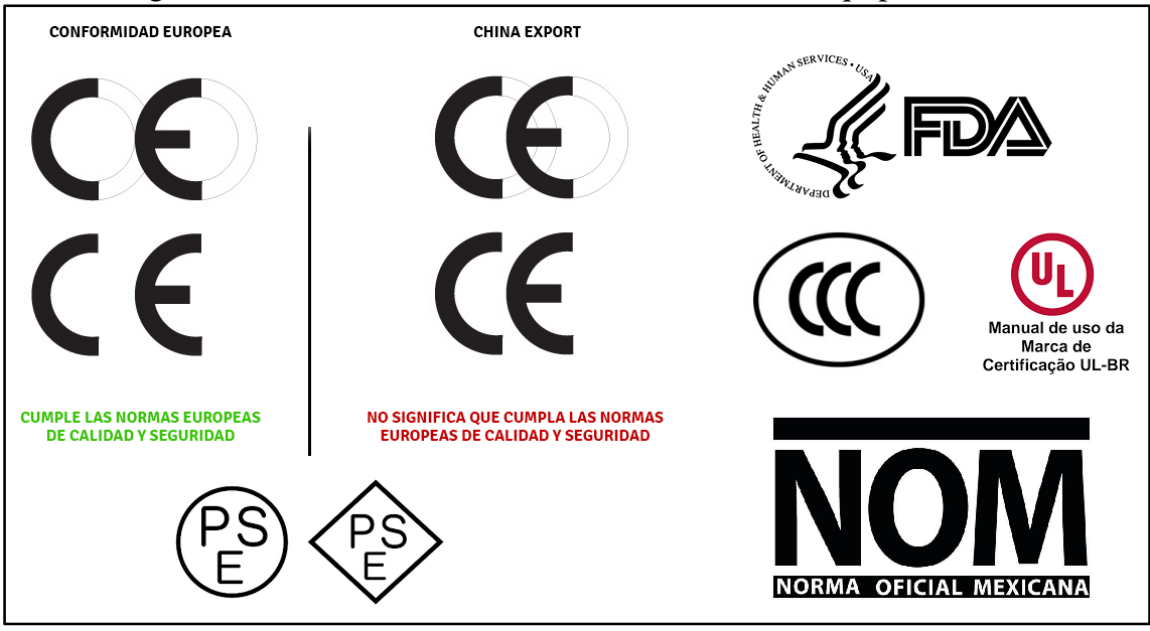

\section{MATERIALES Y MÉTODOS.}

Se aporta al trabajó con un análisis descriptivo-explicativo. El presente estudio se circunscribe como un diseño de investigación de tipo documental orientada a conocer el aporte de la dinámica empleada por la tecnología de la contratación pública electrónica implementada desde el año 2008 a través de la Ley Orgánica de Contratación pública y su Reglamento y el comportamiento de las entidades contratantes; estableciendo como objeto de estudio el IESS Hospital General Machala y servicios agrupados y los beneficios en atenciones médicas de sus pacientes mediante el incremento de la facturación interna de la Unidad Médica. El enfoque a su vez cualitativo sin embargo se apoya en cifras para explicar los beneficios y bondades de la utilización de herramientas de Gestión TI.

Se toma como evidencias de la investigación el portal de compras públicas como herramienta de gestión, donde se encuentran reportados los procesos de contratación en sus distintas modalidades y complejidades de selección de ítems, así como la utilización de tablas para una comparación de varios años entre el 2010 al 2015 de las adquisiciones de equipos médicos, donde se aprecia el aumento significativo en dólares de los servicios hospitalarios en relación a la facturación y demanda de la población afiliada.

\section{DISCUSIÓN DE RESULTADOS}

Los datos e información suministrada en el presente trabajo de investigación se encuentran registradas en el portal de compras públicas en la sección consulta de procesos de entidades contratantes. Figura 5. Información que puede ser difundida para cualquier análisis como pliegos y especificaciones técnicas, necesarias para dar inicio a cualquier proceso de contratación tienen un cronograma en el portal de compras públicas, que comprenden la vigencia de la oferta, el plazo de entrega, así también fechas en las que pueden interactuar las empresas contratantes y las contratistas, 
hasta la fecha de adjudicación dependiendo de la modalidad elegida y si se trata de un bien o un servicio a contratar. Tabla número 2.

Figura 5. Consulta de Procesos Portal de Compras Públicas COTBS-IESS-HM-01-2010

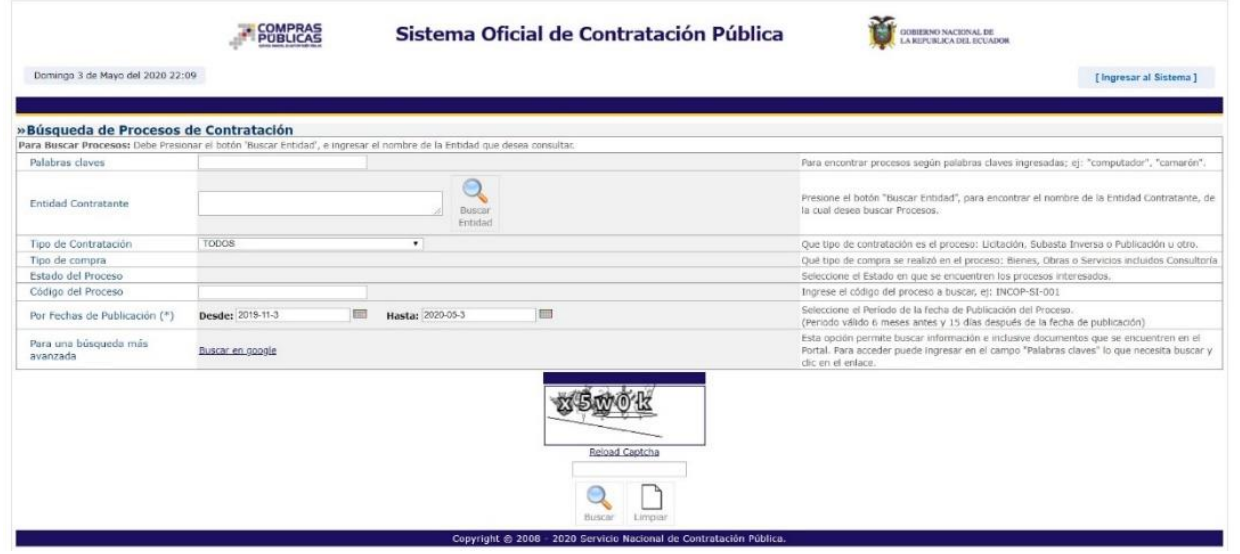

Tabla 2: Ejemplo de fechas de publicación de proceso COTBS-IESS-HM-01-2010

\begin{tabular}{|l|l|l|}
\hline Fecha de Publicación & $9 / 7 / 201010: 00$ & $\begin{array}{l}\text { Indicar la fecha real en la cual desea publicar } \\
\text { el Proceso. }\end{array}$ \\
\hline $\begin{array}{l}\text { Fecha Límite de } \\
\text { Preguntas }\end{array}$ & $\begin{array}{l}14 / 7 / 2010 \\
16: 00\end{array}$ & $\begin{array}{l}\text { Fecha máxima para solicitar aclaraciones } \\
\text { respecto al Proceso de Contratación. }\end{array}$ \\
\hline $\begin{array}{l}\text { Fecha Límite de } \\
\text { Respuestas }\end{array}$ & $\begin{array}{l}16 / 7 / 2010 \\
16: 00\end{array}$ & $\begin{array}{l}\text { Fecha máxima para solventar cualquier } \\
\text { inquietud relacionada al Proceso de } \\
\text { Contratación. }\end{array}$ \\
\hline $\begin{array}{l}\text { Fecha Límite de } \\
\text { Propuestas }\end{array}$ & $\begin{array}{l}19 / 7 / 2010 \\
16: 00\end{array}$ & Fecha máxima para la entrega de propuestas. \\
\hline $\begin{array}{l}\text { Fecha Apertura de } \\
\text { Ofertas }\end{array}$ & $\begin{array}{l}20 / 7 / 2010 \\
12: 00\end{array}$ & $\begin{array}{l}\text { Fecha para la apertura de los sobres de las } \\
\text { ofertas. }\end{array}$ \\
\hline $\begin{array}{l}\text { Fecha Límite solicitar } \\
\text { Convalidación }\end{array}$ & $23 / 7 / 2010$ & $\begin{array}{l}\text { Fecha máxima para que la Entidad notifique } \\
\text { los errores de forma en las ofertas. }\end{array}$ \\
\hline $\begin{array}{l}\text { Fecha Límite respuesta } \\
\text { Convalidación }\end{array}$ & $\begin{array}{l}27 / 7 / 2010 \\
11: 30\end{array}$ & $\begin{array}{l}\text { Fecha máxima para respuestas de } \\
\text { Convalidación de Errores. }\end{array}$ \\
\hline $\begin{array}{l}\text { Fecha Estimada de } \\
\text { Adjudicación }\end{array}$ & $\begin{array}{l}27 / 7 / 2010 \\
16: 00\end{array}$ & $\begin{array}{l}\text { Fecha estimada para la Adjudicación de la } \\
\text { compra. }\end{array}$ \\
\hline
\end{tabular}

Fuente: Portal de Compras Públicas

Elaborado por: Propia de los Autores

La modalidad o procedimiento adoptado por la Unidad de salud más común durante el periodo comprendido al año 2010 al 2015 fue la subasta inversa electrónica para la adquisición de equipos médicos utilizado 25 veces de 51 procesos investigados que ascienden a un precio adjudicado de USD 2184,207.03. Cuando la modalidad de subasta inversa fue imposible de aplicar o se declaró en estado desierto ciertos procesos, se utilizó el procedimiento de cotización solo en dos ocasiones en distintos procesos por un monto total de USD 229,486.00 que representa un $11 \%$ del precio adjudicado de todos los equipos médicos adquiridos mediante el portal. Este procedimiento de cotización se aplicó exclusivamente al 
Tabla 3: Ítems declarados adjudicados en proceso COTBS-IESS-HM-01-2010.

\begin{tabular}{|c|c|c|c|c|c|}
\hline Categoría & Descripción Bien/Obra/Servicio & $\begin{array}{c}\text { Cantidad } \\
\text { Adjudicada }\end{array}$ & $\begin{array}{c}\text { Precio } \\
\text { Unitario }\end{array}$ & Subtotal & Estado \\
\hline 481200119 & $\begin{array}{l}\text { EQUIPO DE ULTRASONIDO, } \\
\text { APLICACION AVANZADA PARA } \\
\text { CUERPO ENTERO, 3D, 4D } \\
\text { FRECUENCIA ENTRE 1-3 MHZ QUE } \\
\text { SEA PULSATIL CONTINUO } \\
\text { TAMAÑO 5CM². }\end{array}$ & 3 & $\$ 1.670,00$ & $\$ 5.010,00$ & Adjudicado \\
\hline 481200119 & $\begin{array}{l}\text { EQUIPO DE ULTRASONIDO, } \\
\text { APLICACION AVANZADA PARA } \\
\text { CUERPO ENTERO, 3D, 4D } \\
\text { FRECUENCIA ENTRE 1-3 MHZ QUE } \\
\text { SEA PULSATIL CONTINUO } \\
\text { TAMAÑO } 2 \text { CM}^{2} .\end{array}$ & 2 & $\$ 1.670,00$ & $\$ 3.340,00$ & Adjudicado \\
\hline 481200119 & $\begin{array}{l}\text { EQUIPO DE ULTRASONIDO, } \\
\text { APLICACION AVANZADA PARA } \\
\text { CUERPO ENTERO, 3D, 4D } \\
\text { FRECUENCIA ENTRE 1-3 MHZ QUE } \\
\text { SEA PULSATIL CONTINUO } \\
\text { TAMAÑO 10CM². }\end{array}$ & 3 & $\$ 1.670,00$ & $\$ 5.010,00$ & Adjudicado \\
\hline 4812001114 & $\begin{array}{l}\text { EQUIPO LASER TERAPIA EQUIPO } \\
\text { LASER ROJO E INFRAROJO MEDIDA } \\
830 \text { NM ULTIMA GENERACION } \\
\text { AJUSTES DE PARAMETROS } \\
\end{array}$ & 8 & $\$ 1.970,00$ & $\$ 15.760,00$ & Adjudicado \\
\hline 4812001113 & $\begin{array}{l}\text { ELECTROESTIMULADOR } \\
\text { NEUROMUSCULAR TIPO DE } \\
\text { CORRIENTE: GALVANICA } \\
\text { INTERRUMPIDA, INTERFERANCIAL, } \\
\text { VIADINAMICAS, TENS. } \\
\end{array}$ & 8 & $\$ 2.982,00$ & $\$ 23.856,00$ & Adjudicado \\
\hline 4818001129 & $\begin{array}{l}\text { RUEDA DE HOMBRO EJERCICIO } \\
\text { MAGNETICA CON LONG DE BRAZO } \\
\text { AJUSTABLE } 33 \text { A 55 CM PANTALLA }\end{array}$ & 2 & $\$ 475,00$ & $\$ 950,00$ & Adjudicado \\
\hline 4815009169 & $\begin{array}{l}\text { GENERADOR DE CAMPOS } \\
\text { MAGNETICOS PULSATIL } \\
\text { (MAGNETO) FR: 50-10 HZ TRENES } \\
\text { DE IMPULSO }\end{array}$ & 6 & $\$ 8.176,00$ & $\$ 49.056,00$ & Adjudicado \\
\hline 4815009169 & $\begin{array}{l}\text { TANQUE DE COMPRESAS } \\
\text { QUIMICAS CALIENTES DE } 24 \text { UNDS } \\
\text { (UNIDADES CALEFACCION } \\
\text { MOVILES) }\end{array}$ & 3 & $\$ 3.148,00$ & $\$ 9.444,00$ & Adjudicado \\
\hline 4815009169 & $\begin{array}{l}\text { TANQUE DE COMPRESAS } \\
\text { QUIMICAS CALIENTES DE } 12 \text { UNDS } \\
\text { (UNIDADES CALEFACCION } \\
\text { MOVILES) }\end{array}$ & 1 & $\$ 1.970,00$ & $\$ 1.970,00$ & Adjudicado \\
\hline
\end{tabular}




\begin{tabular}{|c|c|c|c|c|c|}
\hline 4815009169 & $\begin{array}{l}\text { TANQUE DE COMPRESAS } \\
\text { QUIMICAS FRIAS DE } 12 \text { UNDS } \\
\text { (UNIDADES MOVILES) TIPO } \\
\text { MEDICO } \\
\end{array}$ & 4 & $\$ 3.800,00$ & $\$ 15.200,00$ & Adjudicado \\
\hline 4815009169 & $\begin{array}{l}\text { EQUIPO DE TRACCION CERVICAL } \\
\text { LUMBAR DE ULTIMA GENERACION } \\
\text { CICLICA ESTATICA INTERMI } \\
\end{array}$ & 2 & $\$ 4.400,00$ & $\$ 8.800,00$ & Adjudicado \\
\hline 4815009169 & $\begin{array}{l}\text { TABLA (MESA) DE CUADRICEPS } \\
\text { PROFESIONAL FACIL CAMBIO LOS } \\
\text { PATRONES DE RESISTENCIA }\end{array}$ & 2 & $\$ 1.700,00$ & $\$ 3.400,00$ & Adjudicado \\
\hline 4815009169 & $\begin{array}{l}\text { PARALELAS GRADUABLES CON } \\
\text { PLATAFORMA ANTIDESLIZANTE }\end{array}$ & 3 & $\$ 800,00$ & $\$ 2.400,00$ & Adjudicado \\
\hline 4815009169 & $\begin{array}{l}\text { CAMINADORA MECANICA NO } \\
\text { ELECTRICA PARA ADULTOS } \\
\text { BARRA DE MANOS CON } \\
\text { VELOCIMETRO } \\
\end{array}$ & 1 & $\$ 1.200,00$ & $\$ 1.200,00$ & Adjudicado \\
\hline 4815009169 & $\begin{array}{l}\text { GONIOMETRO PLASTICO } \\
\text { RESISTENTE DE } 360^{\circ} \text { PEQUEÑO DE } \\
15 \text { CM } \\
\end{array}$ & 1 & $\$ 20,00$ & $\$ 20,00$ & Adjudicado \\
\hline 4815009170 & $\begin{array}{l}\text { GONIOMETRO PLASTICO } \\
\text { RESISTENTE DE } 360^{\circ} \text { PEQUEÑO DE } \\
20 \text { CM }\end{array}$ & 4 & $\$ 20,00$ & $\$ 80,00$ & Adjudicado \\
\hline 4815009169 & $\begin{array}{l}\text { GONIOMETRO PLASTICO } \\
\text { RESISTENTE DE } 360^{\circ} \text { PEQUEÑO DE } \\
30 \mathrm{CM}\end{array}$ & 1 & $\$ 20,00$ & $\$ 20,00$ & Adjudicado \\
\hline \multicolumn{4}{|c|}{$\begin{array}{l}\text { TOTAL } \\
\end{array}$} & \multicolumn{2}{|c|}{$\$ 145.516,00$} \\
\hline
\end{tabular}

Fuente: Portal de Compras Públicas

Elaborado por: Propia de los Autores

Los parámetros para evaluar equipos médicos por parte de los profesionales del área médica y especialistas son bastante exigentes por la magnitud de la responsabilidad en cuanto a salvaguardar la vida de los pacientes, la comisión técnica y subcomisiones, se dieron la ardua tarea de revisar cada ítem examinando por modalidad simple (si cumple o no cumple) lo establecido en los pliegos y ficha técnica de especificaciones, consumando lo dispuesto en el cronograma de adquisición. De esta manera se registran en el portal de compras públicas todas las novedades a través de un acta de calificación de parámetros denominada resumen de calificaciones. Tabla 4.

Tabla 4: Parámetros de Calificación COTBS-IESS-HM-01-2010.

\begin{tabular}{|l|r|}
\hline Parámetros & Porcentaje \\
\hline Experiencia & $5 \%$ \\
\hline Plazo de Entrega & $3 \%$ \\
\hline Oferta Económica & $40 \%$ \\
\hline Certificados de calidad de bienes & $2 \%$ \\
\hline Cumplimiento Especificaciones & $15 \%$ \\
\hline Índices Financieros & $5 \%$ \\
\hline Personal Técnico & $3 \%$ \\
\hline Participación Nacional & $10 \%$ \\
\hline Mypes Nacionales & $10 \%$ \\
\hline Mypes - Participación Local & $5 \%$ \\
\hline
\end{tabular}




\begin{tabular}{|l|r|}
$\begin{array}{l}\text { Bonificación por ser favorecido } \\
\text { en el Sorteo }\end{array}$ & $2 \%$ \\
\hline TOTAL & $\mathbf{1 0 0 \%}$ \\
\hline
\end{tabular}

Fuente: Portal de compras públicas

Elaborado por: Los autores

Haciendo otro análisis por servicios o áreas, el servicio de hospitalización, quirófanos, UCI Neonatal y UCI de adultos fue la que mayor inversión en compras de equipamiento médico en el hospital demando durante los años descritos anteriormente con un aporte de USD 889,753.01, equipos que no se hubieran adquirido sino fuera por la intervención del portal de compras que dinamizó el proceso de una manera eficiente para que en el año 2011 el Hospital del IESS de la ciudad de Machala cuente con su primera Unidad de Cuidado Intensivos para adultos que inicio con apenas una central de monitoreo, cuatro camas de uci hospitalarias con su respectivo monitor multiparámetros, y cuatro respiradores volumétricos que han logrado salvar la vida de cientos de afiliados desde esa fecha y mediante la construcción del nuevo hospital que fue inaugurado el 15 de febrero de 2017 ha incrementado el número de camas y su importancia trascendental en los tiempos de pandemia por COVID-19. Respecto a los totales y números de procesos por distintas modalidades se pueden apreciar en la Tabla 5.

Tabla 5. Análisis de Adquisición de Equipos médicos destinados al Servicio de Hospitalización, UCI

\begin{tabular}{|c|c|c|c|c|c|c|c|}
\hline \multicolumn{8}{|c|}{ PROCESOS DE ADQUISICION DE EQUIPOS MEDICOS IESS HOSPITAL GENERAL MACHALA } \\
\hline SERVICIOS & AÑOS: & 2.010 & 2.011 & $\begin{array}{c}2.01 \\
2\end{array}$ & $\begin{array}{c}2.01 \\
3\end{array}$ & 2.014 & 2.015 \\
\hline $\begin{array}{l}\text { QUIROFANO Y } \\
\text { HOSPITALIZACION, } \\
\text { UCI }\end{array}$ & PROCESO & $53.290,92$ & 275.123,94 & $\mathbf{0 , 0 0}$ & $\mathbf{0 , 0 0}$ & 141.126,20 & 420.211,95 \\
\hline $\begin{array}{l}\text { ADQ. EQUIPOS MEDICOS } \\
\text { PARA USO DE LA UCI } \\
\text { DEL SERVICIO DE } \\
\text { NEONATOLOGIA Y } \\
\text { PEDIAT. }\end{array}$ & SUBASTA & $25.882,50$ & - & - & - & - & - \\
\hline $\begin{array}{l}\text { ADQ. EQUIPO MEDICO } \\
\text { CAMARA DIGITAL DE } \\
\text { VIDEOCIRUGIA DE TRES } \\
\text { CHIPS, PARA } \\
\text { LAPROSCOPIO STRYKER }\end{array}$ & $\begin{array}{l}\text { REGIMEN } \\
\text { ESPECIAL }\end{array}$ & $22.620,00$ & - & - & - & - & - \\
\hline $\begin{array}{l}\text { LAMPARA XENON } 150 \\
\text { W PARA LA FUENTE } \\
\text { LUZ }\end{array}$ & $\begin{array}{l}\text { I. } \\
\text { CUANTIA }\end{array}$ & $2.000,00$ & - & - & - & - & - \\
\hline $\begin{array}{l}\text { NEUROESTIMULADOR } \\
\text { NERVIOS PERIFERICOS }\end{array}$ & $\begin{array}{l}\text { I. } \\
\text { CUANTIA }\end{array}$ & $2.788,42$ & - & - & - & - & - \\
\hline $\begin{array}{l}\text { EQUIPOS MEDICOS } \\
\text { PARA LA } \\
\text { IMPLEMENTACION DE } \\
\text { UCI. }\end{array}$ & SUBASTA & - & $105.876,55$ & - & - & - & - \\
\hline $\begin{array}{l}\text { ADQ. EQUIPOS MEDICOS } \\
\text { DE UCI CAMAS } \\
\text { HOSPITALARIAS }\end{array}$ & SUBASTA & - & $29.190,00$ & - & - & - & - \\
\hline
\end{tabular}




\begin{tabular}{|c|c|c|c|c|c|c|c|}
\hline $\begin{array}{l}\text { ADQ. EQUIPOS MEDICOS } \\
\text { AREA DE } \\
\text { NEUROCIRUGIA } \\
\text { CRANEOTOMO Y } \\
\text { FRONTOLUX }\end{array}$ & SUBASTA & - & $23.248,39$ & - & - & - & \\
\hline $\begin{array}{l}\text { ADQ. DE EQUIPO } \\
\text { MEDICO } \\
\text { ESTERILIZADORA A } \\
\text { BASE DE TECNOLOGIA } \\
\text { DE PEROXIDO DE } \\
\text { HIDROGENO }\end{array}$ & SUBASTA & - & $87.950,00$ & 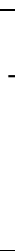 & - & - & \\
\hline $\begin{array}{l}\text { COMPRA LAMPARA DE } \\
\text { XENON }\end{array}$ & $\begin{array}{l}\text { I. } \\
\text { CUANTIA }\end{array}$ & - & $1.950,00$ & . & - & - & - \\
\hline $\begin{array}{l}\text { ADQ. EQUIPOS DE } \\
\text { COMPUTO EN SERVICIO } \\
\text { DE HOSPITALIZACION }\end{array}$ & SUBASTA & - & $26.909,00$ & - & - & - & \\
\hline $\begin{array}{l}\text { ADQ, ELECTROBISTURI } \\
\text { MARCA ZERONE }\end{array}$ & SUBASTA & - & - & - & - & $6.400,00$ & - \\
\hline $\begin{array}{l}\text { ADQ. } 3 \text { MONITORES DE } \\
\text { SIGNOS VITALES }\end{array}$ & SUBASTA & - & - & . & - & $60.945,00$ & - \\
\hline $\begin{array}{l}\text { ADQ. } 2 \text { MONITORES DE } \\
\text { SIGNOS VITALES }\end{array}$ & SUBASTA & - & - & - & - & $40.630,00$ & - \\
\hline $\begin{array}{l}\text { ADQ. } 12 \text { MONITORES DE } \\
\text { SIGNOS VITALES }\end{array}$ & SUBASTA & - & - & . & - & $33.151,20$ & - \\
\hline $\begin{array}{l}\text { AQD. } 10 \text { MONITORES } \\
\text { MULTIPARAMETROS } \\
\text { BASICO }\end{array}$ & $\begin{array}{l}\text { C. } \\
\text { ELECTRÓN } \\
\text { ICO }\end{array}$ & - & - & - & - & - & $30.941,12$ \\
\hline $\begin{array}{l}\text { ADQ. TRES } \\
\text { ELECTROCARDIOGRAF } \\
\text { OS DE SEIS CANALES }\end{array}$ & $\begin{array}{l}\text { I. } \\
\text { CUANTIA }\end{array}$ & - & - & - & - & - & $6.500,00$ \\
\hline $\begin{array}{l}\text { EQUIPO PARA COCHE } \\
\text { DE VIA AEREA }\end{array}$ & $\begin{array}{l}\text { I. } \\
\text { CUANTIA }\end{array}$ & - & - & - & - & - & $3.760,00$ \\
\hline $\begin{array}{l}\text { ADQ. FUENTE LUZ LED } \\
\text { L9000 }\end{array}$ & SUBASTA & - & - & ב & - & - & $8.500,00$ \\
\hline $\begin{array}{l}\text { ADQ, INSUFLADOR DE } \\
\text { CO2 PARA TORRE }\end{array}$ & SUBASTA & - & - & - & - & - & $10.000,00$ \\
\hline $\begin{array}{l}\text { ADQ, VENTILADOR CO2, } \\
\text { UNIDAD ELECTRONICA }\end{array}$ & SUBASTA & - & - & - & - & - & $41.448,50$ \\
\hline $\begin{array}{l}\text { ADQ. INCUBADORA DE } \\
\text { TRANSPORTE Y } \\
\text { MONITOR }\end{array}$ & SUBASTA & - & - & . & - & - & $34.196,00$ \\
\hline $\begin{array}{l}\text { ADQ. 10 CAMAS } \\
\text { ELECTRICAS } \\
\text { MULTIPLES- }\end{array}$ & SUBASTA & - & - & - & - & - & $57.999,88$ \\
\hline $\begin{array}{l}\text { ADQ. DOS EQUIPOS } \\
\text { DESFIBRILIDORES CON } \\
\text { MARCAPASOS }\end{array}$ & SUBASTA & - & - & - & - & - & $22.800,00$ \\
\hline $\begin{array}{l}\text { ADQ. TRES LAMPARAS } \\
\text { CIELITICA SERIE } 123- \\
\text { ACT }\end{array}$ & SUBASTA & - & - & - & - & - & $72.675,00$ \\
\hline $\begin{array}{l}\text { ADQ, AUTOCLAVE } \\
\text { SEMIAUTOMATICA, } \\
\text { LAMPARA }\end{array}$ & $\begin{array}{l}\text { I. } \\
\text { CUANTIA }\end{array}$ & - & - & - & - & - & $3.720,46$ \\
\hline $\begin{array}{l}\text { ADQ. UNA MAQUINA DE } \\
\text { ANESTESIA, MESA }\end{array}$ & SUBASTA & - & - & - & - & - & $113.210,99$ \\
\hline $\begin{array}{l}\text { ADQ. } 3 \\
\text { ELECTROBISTURI }\end{array}$ & $\begin{array}{l}\text { I. } \\
\text { CUANTIA }\end{array}$ & - & - & - & - & - & $7.200,00$ \\
\hline $\begin{array}{l}\text { ADQ. UN MONITOR DE } \\
\text { PRO UNDDAD HIPNOTI) }\end{array}$ & $\begin{array}{l}\text { I. } \\
\text { CUANTIA }\end{array}$ & - & - & - & - & - & $7.260,00$ \\
\hline
\end{tabular}


Fuente: Portal de compras públicas

Elaborado por: Los autores

Es necesario analizar que las atenciones del servicio de rehabilitación y terapia física del IESS Hospital de Machala se incrementaron en su demanda, pero de una manera ordenada al haberse incorporado más equipos médicos y en consecuencia el aumento de talento humano en esa área. Todo gracias a la gestión realizada mediante la herramienta del portal de compras públicas por profesionales de la misma unidad médica con una adquisición de equipos por el valor de USD 402,406.00 durante cinco años. El descompresor lumbar ha permitido a ciento de pacientes con problemas lumbares y hernias discales realizar un tratamiento que alivia estas dolencias. De la misma manera las ondas de choque permiten brindar tratamientos a pacientes con problemas de dolor de hombro, espolones y principalmente en las articulaciones al aplicarse una dosis de disparos medicinales mediante la fuerza empleada por un bar de presión $(1$ bar $=100.000 \mathrm{~Pa}=1,01972 \mathrm{kgf} / \mathrm{cm} 2)$. Respecto a los totales y números de procesos por distintas modalidades se pueden apreciar en la Tabla 6.

Tabla 6. Análisis de Adquisición de Equipos médicos destinados al Servicio de Fisioterapia

\begin{tabular}{|c|c|c|c|c|c|c|c|}
\hline \multicolumn{8}{|c|}{$\begin{array}{l}\text { PROCESOS DE ADQUISICION DE EQUIPOS MEDICOS IESS HOSPITAL GENERAL } \\
\text { MACHALA }\end{array}$} \\
\hline SERVICIOS & AÑNOS: & 2.010 & 2.011 & 2.012 & 2.013 & 2.014 & 2.015 \\
\hline $\begin{array}{l}\text { REHABILITACION Y } \\
\text { TERAPIA FISICA }\end{array}$ & PROCESO & $307.516,00$ & $88.410,00$ & $\mathbf{0 , 0 0}$ & $\mathbf{0 , 0 0}$ & $6.480,00$ & $\mathbf{0 , 0 0}$ \\
\hline $\begin{array}{l}\text { ADQ. DE EQUIPO } \\
\text { MEDICO } \\
\text { DESCOMPRESOR } \\
\text { LUMBAR. }\end{array}$ & SUBASTA & $162.000,00$ & - & - & - & - & - \\
\hline $\begin{array}{l}\text { ADQ. DE EQUIPOS } \\
\text { MEDICOS PARA } \\
\text { FISIOTERAPIA COTBS- } \\
\text { IESS-HM-01-2010 }\end{array}$ & COTIZACIÓN & $145.516,00$ & - & - & - & - & - \\
\hline $\begin{array}{l}\text { ADQ. EQUIPOS DE } \\
\text { ONDAS DE CHOQUE }\end{array}$ & COTIZACIÓN & - & $83.970,00$ & - & - & - & - \\
\hline $\begin{array}{l}\text { EQUIPOS DE } \\
\text { FISIOTERAPIA (DOS } \\
\text { VIBRADORES) } \\
\end{array}$ & I. CUANTIA & - & $4.440,00$ & - & - & - & - \\
\hline $\begin{array}{l}\text { POR COMPRA DE } \\
\text { CORTADORA DE YESO } \\
\text { MARCA EQUIMEDICA } \\
\text { SRL }\end{array}$ & I. CUANTIA & - & - & - & - & $1.480,00$ & - \\
\hline $\begin{array}{l}\text { ADQ. LAMPARA DE } \\
\text { FOTOTERAPIA }\end{array}$ & SUBASTA & - & - & - & - & $5.000,00$ & - \\
\hline
\end{tabular}

Fuente: Portal de compras públicas

Elaborado por: Los autores

Además, es necesario identificar el equipamiento médico dotado al servicio de consulta externa en el IESS Hospital de Machala cuya inversión en equipos adquiridos durante los cinco años analizados fue en razón de USD 875,951 constituyéndose en un 40\% de todo el presupuesto empleado en la unidad 
médica en las adquisiciones de cinco años, lo que constituye una buena gestión administrativa y eficiente uso del portal de compras públicas como un mecanismo de contratación pública dinámico y eficiente. Se dotó de equipos médicos a las áreas críticas de atención como gastroenterología donde siempre existe una gran demanda de este servicio y en la de oftalmología para realizar exámenes de mediciones oculares para la población afiliada. Respecto a los totales y números de procesos por distintas modalidades se pueden apreciar en la Tabla 7.

Tabla 7. Análisis de Adquisición de Equipos médicos destinados al Servicio de Consulta externa

\begin{tabular}{|c|c|c|c|c|c|c|c|}
\hline \multicolumn{8}{|c|}{ PROCESOS DE ADQUISICION DE EQUIPOS MEDICOS IESS HOSPITAL GENERAL MACHALA } \\
\hline SERVICIOS & AÑN: & 2.010 & 2.011 & 2.012 & 2.013 & 2.014 & 2.015 \\
\hline $\begin{array}{l}\text { CONSULTA EXTERNA, } \\
\text { GASTROENTEROLOGÍA, } \\
\text { CARDIOLOGIA, } \\
\text { MEDICNA INTERNA }\end{array}$ & PROCESO & 13.974,00 & $50.967,00$ & 7.889,00 & $290.392,00$ & 232.730,00 & 279.999,00 \\
\hline $\begin{array}{l}\text { ELECTROCARDIOGRAFO } \\
\text { DE } 12 \text { CANALES CON } \\
\text { INTERPRETACION } \\
\text { MODELO ATY-2 PLUS S/N } \\
029.01929 .\end{array}$ & SUBASTA & $6.950,00$ & - & - & - & - & - \\
\hline $\begin{array}{l}\text { ELECTROCARDIOGRAFO } \\
\text { SCHILLER DE } 6 \text { CANALES } \\
\text { CARDIVIT AT Y 2N PLUS }\end{array}$ & I. CUANTIA & $3.500,00$ & - & - & - & - & - \\
\hline $\begin{array}{l}\text { ADQ. EQUIPO MEDICO } \\
\text { MONITOR MATERNO } \\
\text { FETAL PARA EL AREA } \\
\text { DE OBSTETRICIA }\end{array}$ & I. CUANTIA & $3.524,00$ & - & - & - & - & - \\
\hline $\begin{array}{l}\text { EQUIPOS MEDICOS PARA } \\
\text { EL AREA DE } \\
\text { CARDIOLOGIA }\end{array}$ & SUBASTA & - & $39.468,00$ & - & - & - & - \\
\hline $\begin{array}{l}\text { ADQ. DE } 5 \text { SET DE } \\
\text { DIAGNOSTICO } \\
\text { INTEGRADOS DE PARED }\end{array}$ & SUBASTA & - & $11.499,00$ & - & - & - & - \\
\hline $\begin{array}{l}\text { ADQ. DE UN } \\
\text { ELECTROCARDIOGRAFO }\end{array}$ & I. CUANTIA & - & - & $3.555,00$ & - & - & - \\
\hline $\begin{array}{l}\text { ADQ. DE DOS BALANZAS } \\
\text { PEDIATRICAS }\end{array}$ & I. CUANTIA & - & - & $2.134,00$ & - & - & - \\
\hline $\begin{array}{l}\text { ADQ. OXIMETRO DE } \\
\text { PULSO }\end{array}$ & I. CUANTIA & - & - & $2.200,00$ & - & - & - \\
\hline $\begin{array}{l}\text { ADQ, EQUIPO } \\
\text { OFTALMOLOGICO } \\
\text { DESDE NIVEL CENTRAL }\end{array}$ & SUBASTA & - & - & - & $290.392,00$ & - & - \\
\hline $\begin{array}{l}\text { POR COMPRA DE DOS } \\
\text { MONITORES FETALES } \\
\text { PORTATILES }\end{array}$ & I. CUANTIA & - & - & - & - & $1.230,00$ & - \\
\hline $\begin{array}{l}\text { ADQ. EQUIPO COMPLETO } \\
\text { GASTROENTEROLOGIA }\end{array}$ & SUBASTA & - & - & - & - & $231.500,00$ & - \\
\hline
\end{tabular}




\begin{tabular}{|l|l|l|l|l|l|l|l|}
\hline $\begin{array}{l}\text { ADQ, SISTEMA LASER DE } \\
\text { ALTA POTENCIA }\end{array}$ & $\begin{array}{l}\text { R. } \\
\text { ESPECIAL }\end{array}$ & - & - & - & & - & - \\
\hline
\end{tabular}

Fuente: Portal de compras públicas

Elaborado por: Los autores

Las herramientas de gestión de las tecnologías de la información como el portal de compras aceleran los procesos de compras y adquisiciones de equipos médicos bajo estándares muy altos utilizando el escenario virtual para contratar bajo diferentes modalidades establecidas en la LOSNCP. A continuación, como un resumen de las adquisiciones del año 2010 al 2015, se aprecia un cuadro consolidado de adquisiciones por servicio, puesto que en los últimos cuatro años al contar con un hospital totalmente nuevo no se ha necesitado más inversiones en equipamiento médico. Para ver el resumen de adquisiciones por servicio, puede apreciarse la información de la Tabla 8.

Tabla 8: Consolidado de adquisiciones por servicios de salud IESS Hospital General Machala

\begin{tabular}{|l|c|c|c|c|c|c|c|}
\hline \multicolumn{7}{|c|}{ RESUMEN DE PROCESOS DE ADQUISICION DE EQUIPOS MEDICOS POR SERVICIOS } \\
\hline SERVICIOS & $\mathbf{2 . 0 1 0}$ & $\mathbf{2 . 0 1 1}$ & $\mathbf{2 . 0 1 2}$ & $\mathbf{2 . 0 1 3}$ & $\mathbf{2 . 0 1 4}$ & $\mathbf{2 . 0 1 5}$ & TOTAL \\
\hline $\begin{array}{l}\text { TOTAL IESS } \\
\text { HOSPITAL DE } \\
\text { MACHALA }\end{array}$ & $\mathbf{3 7 4 . 7 8 0 , 9 2}$ & $\mathbf{4 1 6 . 1 8 2 , 4 4}$ & $\mathbf{7 . 8 8 9 , 0 0}$ & $\mathbf{2 9 4 . 2 9 1 , 7 8}$ & $\mathbf{3 8 0 . 3 3 6 , 2 0}$ & $\mathbf{7 1 0 . 7 2 6 , 6 9}$ & $\mathbf{2 . 1 8 4 . 2 0 7 , 0 3}$ \\
\hline $\begin{array}{l}\text { REHABILITACION Y } \\
\text { TERAPIA FISICA }\end{array}$ & $307.516,00$ & $88.410,00$ & 0,00 & 0,00 & $6.480,00$ & 0,00 & $402.406,00$ \\
\hline $\begin{array}{l}\text { QUIROFANO Y } \\
\text { HOSPITALIZACION, UCI }\end{array}$ & $53.290,92$ & $275.123,94$ & 0,00 & 0,00 & $141.126,20$ & $420.211,95$ & $889.753,01$ \\
\hline $\begin{array}{l}\text { CONSULTA EXTERNA, } \\
\text { GASTROENTEROLOGİA, } \\
\text { CARDIOLOGIA, } \\
\text { MEDICNA INTERNA }\end{array}$ & $13.974,00$ & $50.967,00$ & $7.889,00$ & $290.392,00$ & $232.730,00$ & $279.999,00$ & $875.951,00$ \\
\hline $\begin{array}{l}\text { LABORATORIOS DE } \\
\text { RAYOS X, CLINICO Y } \\
\text { PATOLOGIA }\end{array}$ & 0 & & & & & & $10.515,74$ \\
\end{tabular}

Fuente: Portal de compras públicas

Elaborado por: Los autores

Es necesario señalar que en la facturación interna de la unidad de salud todos los servicios que atienden directamente a un paciente son colocados en el Servicio de Consulta Externa aumentando en dólares americanos en el año 2011 por USD 5.488,693.96 a un 33.32\% por un valor de USD 8.231,832.80 en el año 2012 y un 7.75\% de crecimiento respecto del año anterior USD 8.922,960.06, existiendo otros servicios que se reportan mensualmente y consolidado anualmente. Tabla 9. 
Tabla 9. Comparación de la facturación por servicio años 2011-2013 IESS Hospital General Machala.

\begin{tabular}{|c|c|c|c|c|}
\hline CUENTAS & DESCRIPCION & 2011 & 2012 & 2013 \\
\hline 140505020100 & $\begin{array}{l}\text { SERVICIOS MEDICOS AL } \\
\text { SSIF }\end{array}$ & $\begin{array}{l}\$ \\
6.205 .573,07\end{array}$ & $\begin{array}{l} \\
10.635 .388,33\end{array}$ & $\begin{array}{l}\text { \$ } \\
13.049 .578,26\end{array}$ \\
\hline 140505090100 & $\begin{array}{l}\text { ATENCION MED A } \\
\text { JUBILADOS }\end{array}$ & $\begin{array}{l}\$ \\
1.890 .791,44\end{array}$ & $\begin{array}{l}\$ \\
2.782 .755,57\end{array}$ & $\begin{array}{l}\$ \\
3.759 .910,38\end{array}$ \\
\hline 140505090200 & $\begin{array}{l}\text { ATENCION MEDICA } \\
\text { BENEFICIARIOS DE } \\
\text { MONTEPIO }\end{array}$ & $\begin{array}{l}\$ \\
380.920,81\end{array}$ & $\begin{array}{l}\$ \\
396.264,25\end{array}$ & $\begin{array}{l}\$ \\
584.762,33\end{array}$ \\
\hline 140505040100 & $\begin{array}{l}\text { ATENCION MEDICA S } \\
\text { PREST AL SSC }\end{array}$ & $\begin{array}{l}\$ \\
407.780,80\end{array}$ & $\begin{array}{l}\$ \\
601.734,48\end{array}$ & $\begin{array}{l}\$ \\
1.131 .498,74\end{array}$ \\
\hline 140505080100 & $\begin{array}{l}\text { ATENCION MEDICA A } \\
\text { PACIENTES } \\
\text { PARTICULARES }\end{array}$ & $\begin{array}{l}\$ \\
2.373,66\end{array}$ & $\begin{array}{l}\$ \\
37.814,22\end{array}$ & $\begin{array}{l}\$ \\
159.472,97\end{array}$ \\
\hline \multicolumn{2}{|c|}{ SUBTOTAL DEUDOR } & $\begin{array}{l}\$ \\
10.123 .598,83\end{array}$ & $\begin{array}{l}\$ \\
14.453 .956,85\end{array}$ & $\begin{array}{l} \\
18.685 .222,68\end{array}$ \\
\hline 510505020100 & $\begin{array}{l}\text { VENTAS DE MEDICINA } \\
\text { (FARMACIA) }\end{array}$ & $\begin{array}{l}\$ \\
1.346 .385,58\end{array}$ & $\begin{array}{l}\$ \\
2.804 .186,14\end{array}$ & $\begin{array}{l}\$ \\
4.917 .493,93\end{array}$ \\
\hline 510505020200 & $\begin{array}{l}\text { VENTAS DE INSUMOS } \\
\text { MEDICOS (ENFERMERIA) }\end{array}$ & $\begin{array}{l}\$ \\
97.854,86\end{array}$ & $\begin{array}{l}\$ \\
19.074,48\end{array}$ & $\$$ \\
\hline 510505010100 & $\begin{array}{l}\text { VENTAS DE SERVICIO } \\
\text { MEDICO EMERGENCIA }\end{array}$ & $\begin{array}{l}\$ \\
154.130,28\end{array}$ & $\begin{array}{l}\$ \\
404.059,12\end{array}$ & $\begin{array}{l}\$ \\
1.393 .239,10\end{array}$ \\
\hline 510505010200 & $\begin{array}{l}\text { VENTA DE SERVICIO } \\
\text { MEDICO CONSULTA } \\
\text { EXTERNA }\end{array}$ & $\begin{array}{l}\$ \\
5.488 .693,96\end{array}$ & $\begin{array}{l}\$ \\
8.231 .832,80\end{array}$ & $\begin{array}{l}\$ \\
8.922 .960,06\end{array}$ \\
\hline 510505010300 & $\begin{array}{l}\text { VENTA DE SERVICIO } \\
\text { MEDICO } \\
\text { HOSPITALIZACION } \\
\end{array}$ & $\begin{array}{l}\$ \\
812.677,81\end{array}$ & $\begin{array}{l}\$ \\
690.736,51\end{array}$ & $\begin{array}{l}\$ \\
3.451 .529,59\end{array}$ \\
\hline 510505030100 & $\begin{array}{l}\text { VENTAS DE SERVICIOS } \\
\text { ODONTOLOGICOS }\end{array}$ & $\begin{array}{l}\$ \\
81.125,60\end{array}$ & $\begin{array}{l}\$ \\
163.580,21\end{array}$ & $\$$ \\
\hline 510505040100 & $\begin{array}{l}\text { VENTAS DE EXAMENES } \\
\text { DE LABORATORIO }\end{array}$ & $\begin{array}{l}\$ \\
416.188,21\end{array}$ & $\begin{array}{l}\$ \\
1.154 .095,51\end{array}$ & $\$$ \\
\hline 510505040200 & $\begin{array}{l}\text { VENTA DE SERVICIOS } \\
\text { DE RAYOS X }\end{array}$ & $\begin{array}{l}\$ \\
375.183,92\end{array}$ & $\begin{array}{l}\$ \\
986.392,08\end{array}$ & $\$$ \\
\hline \multicolumn{2}{|c|}{ SUBTOTAL ACREEDOR } & $\begin{array}{l}\$ \\
10.123 .599,03\end{array}$ & $\begin{array}{l}\$ \\
14.453 .956,85\end{array}$ & $\begin{array}{l}\$ \$ \\
18.685 .222,68\end{array}$ \\
\hline
\end{tabular}

Fuente: Departamento de Facturación del IESS Hospital General Machala Elaborado por: Propia de los Autores.

Las cantidades de pacientes y valores facturados en Consulta Externa crecieron en los años 2011, 2012 y 2013 desde que todos los ítems adquiridos en el proceso de Cotización fueron instalados a fines del año 2010. Figura 6. 
Figura 6. Gráfico de Crecimiento del Servicio de Consulta externa.

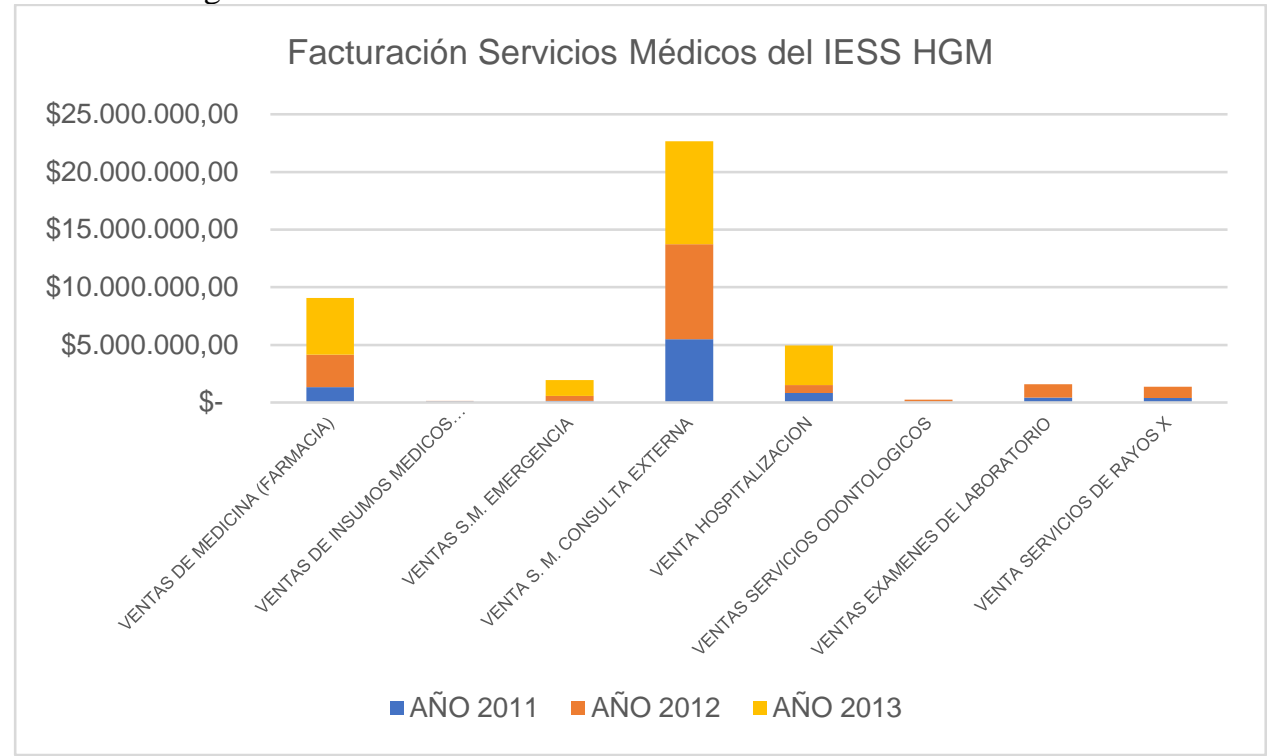

\section{CONCLUSIONES}

El mecanismo tradicional de contratación pública tradicional existente antes del año 2010, hubiera hecho imposible una adquisición realizada por la modalidad de cotización, tanto en plazos como selección de parámetros y transparencia. Definitivamente gracias a las aplicaciones y herramientas tecnológicas como el portal de compras, la contratación pública electrónica es mucho más eficiente en los aspectos descritos y aunque requiere de una extrema planificación de especificaciones técnicas y modelos de pliegos, esto es un parámetro que permite hacer rigurosa la selección de un ítem sea equipo, insumo, dispositivo u otro bien o servicio a contratar por las entidades púbicas con sus respectivas partidas presupuestarias CP, POA y PAC debidamente aprobados y con resoluciones motivadas por las máxima autoridad de la entidad contratante.

Las ventajas de utilizar el portal de compras, permite que la contratación pública electrónica se desarrolle en línea, se concretan en la eficiencia de tiempo, esfuerzo de comisiones y subcomisiones de funcionarios públicos y su aplicación mediante la capacitación continua en el manejo tecnológico de procedimientos normados y medios electrónicos. Los Jubilados, pensionistas y afiliados de la Seguridad Social son los grandes beneficiarios al utilizar servicios de calidad y calidez impulsadas por las autoridades gubernamentales de turno y la planificación en función de las necesidades de la población.

El equipamiento médico en hospitales públicos mediante servicios focalizados en la rehabilitación, hospitalización, consulta externa y laboratorios, favorecen en la recuperación de los pacientes e incrementan la facturación interna de estas unidades de salud como unidades de negocio. El mecanismo de contratación pública electrónica llego para quedarse y mejorar continuamente hasta ser perfectible. Solo está en los profesionales y autoridades utilizarlo de manera eficiente para las entidades contratantes y contratistas para que los exámenes de los organismos de control sean equilibrados y transparentes. 
Está claro que el IESS Hospital General Machala seguirá siendo un referente en salud de la provincia de El Oro, sobre todo desde la construcción del Nuevo Hospital en el año 2016 y su inauguración realizada en el año 2017 donde hoy atiende a un mayor número de pacientes con equipamiento nuevo. La pandemia COVID 19 nos permite aportar la importancia de equipos médicos implementados en la UCI de adultos ha permitido atender a cientos de pacientes desde marzo del 2020 cuando se inició con el primer caso detectado. De ahí la importancia de planificar anualmente las adquisiciones en la herramienta del portal web de compras públicas que ha permitido la adjudicación de manera virtual de insumos y medicamentos para su posterior uso como complemento de aquellos equipos médicos de vanguardia para el beneficio de la población afiliada y en circunstancias críticas a pacientes derivados del sistema de salud público.

\section{Abreviaturas}

IESS: Instituto Ecuatoriano de Seguridad Social del Ecuador; POA: Plan Operativo Anual; PAC: Plan Anual de Compras Públicas; MSP: Ministerio de Salud Pública; MIES: Ministerio de Inclusión Económica y Social; ISSFA: Instituto de Seguridad Social de las Fuerzas Armadas; ISSPOL: Instituto de Seguridad Social de la Policía Nacional; LOSNCP: Ley Orgánica del Sistema Nacional de Contratación Pública. 


\section{REFERENCIAS BIBLIOGRÁFICAS}

Aguirre Ribadeneira, J. (2016). Contratación pública ecuatoriana en el acuerdo comercial con la Unión Europea (Tema Central). FORO. Revista de Derecho, 25, 27-56. https://revistas.uasb.edu.ec/index.php/foro/article/view/461

Barragan Martinez, X., \& Guevara Viejo, F. (2016). El gobierno electrónico en Ecuador / E-Government in Ecuador. Ciencia Unemi, 9(19), 110. https://doi.org/10.29076/issn.2528-7737vol9iss19.2016pp110$127 \mathrm{p}$

Bossano Lomellini, L. (2011). La subasta inversa: un mecanismo de contratación pública eficiente y transparente. Derecho PUCP: Revista de la Facultad de Derecho, 66, 277-285. https://dialnet.unirioja.es/servlet/articulo?codigo $=5085251$

Chinea, O. (2013). Cualificación, acreditación y certificación de la Contratación Pública en América Latina y el Caribe. Revista del CLAD Reforma y Democracia, 211-226. https://www.redalyc.org/articulo.oa?id=357533688007

Delpiazzo, C. (2011). Contratación Pública electrónica en Europa y América Latina - Dialnet. Derecho PUCP: Revista de la Facultad de Derecho, 66, 147-167. https://dialnet.unirioja.es/servlet/articulo?codigo $=5085268$

Ganga Contreras, F., \& Águila Sánchez, M. (2006). LAS TECNOLOGÍAS DE INFORMACIÓN Y COMUNICACIÓN (TIC) Y SU IMPACTO EN EL SISTEMA DE COMPRAS Y CONTRATACIONES PÚBLICAS: El caso de la región de Los Lagos en Chile. Journal of Economics, Finance and Administrative Science, 11(21), 41-67. https://www.redalyc.org/articulo.oa?id=360733601003

Laguado, G. R. (2004). LA CONTRATACIÓN PÚBLICA ELECTRÓNICA EN COLOMBIA | Vniversitas. $\quad$ Vniversitas, 53(108),

https://revistas.javeriana.edu.co/index.php/vnijuri/article/view/14746

Lucio, R., Villacrés, N., \& Henríquez, R. (2011). [The health system of Ecuador]. Salud publica de Mexico, 53 Suppl 2, s177-87. https://doi.org/10.1590/S0036-36342011000800013

Moscoso, A. M. (2014). La transparencia en la Administración Pública del Ecuador. Las compras públicas electrónicas el "cubo de cristal" de la accountability y buen gobierno. Direito Público, 11(56), 186-209. https://doi.org/10.11117/22361766.56.11.2075

Registro Oficial República del Ecuador. (2008, agosto 4). Registro Oficial No. 395. Ley Orgánica del Sistema Nacional de Contratación Pública. https://www.registroficial.gob.ec/index.php/registro-oficialweb/publicaciones/registro-oficial/item/4494-registro-oficial-no-395

Rivas, D., \& Zambrano, M. (2019). PROCEDIMIENTOS DE CONTRATACIÓN PÚBLICA EN LA UNIVERSIDAD TÉCNICA DE MANABÍ. Revista Caribeña de Ciencias Sociales, 2019-08. https://www.eumed.net/rev/caribe/2019/08/contratacion-publica-universidad.html 